\title{
A Novel Single Power Two Circuits Electroslag Remelting with Current Carrying Mould
}

\author{
Yanwu DONG, ${ }^{*}$ Zhouhua JIANG, Haibo CAO, Xiang WANG, Yulong CAO and Dong HOU \\ School of Metallurgy, Northeastern University, Shenyang, Liaoning, 110819 China. \\ (Received on February 3, 2016; accepted on April 13, 2016; J-STAGE Advance published date: June 28, \\ 2016)
}

\begin{abstract}
A novel single power two circuits electroslag remelting (ESR) with current carrying mould is presented in this paper. There are two processes with up power and down power for this technology. Numerical simulation of single power two circuits electroslag remelting process with current carrying mould (ESRSTCCM) as well as conventional ESR process for comparison is carried out in this paper. Results indicate that ESR-STCCM process changes the current circuit. Special current circuit results in different electromagnetic field, fluids flow and thermal field. Molten steel pool is shallower and mushy zone is narrower for ESR-STCCM than conventional ESR process. In addition, the temperature of slag-metal interface for ESRSTCCM with up power is higher than others ESR process investigated in this article that contributes to obtain smooth ingot surface quality. All in all, ESR-STCCM with up power is a promising process for improving the quality of material prone to segregation in comparison with other ESR process, based o the results obtained in this article.
\end{abstract}

KEY WORDS: electroslag; quality control; single power two circuits electroslag remelting process with current carrying mould (ESR-STCCM); molten steel pool; low segregation.

\section{Introduction}

Along the development of electroslag remelting (ESR), current carrying mould had been invented and used in ESR process. Medovar and Tskulenko et al. ${ }^{1,2)}$ and Holzgruber et $a l .{ }^{3)}$ had done an experiment using current carrying mould (a double power two-circuit ESR system). A smooth surface and low element segregation ingot are obtained even at low melting rate by using current carrying mould. Dong et al. ${ }^{4)}$ had manufactured solid ingot using current carrying mould with liquid metal replacing conventional consumable electrode and relevant simulation had been done. Liu et al. ${ }^{5)}$ had melted hollow ingots with T-shape current carrying. As a matter of fact, there are two power supplies in the investigation of Medovar and Tskulenko et al., Holzgruber et al. and Liu et al. It had been found that there is some interference between two powers in circuit in the real experiment.

In this work electroslag remelting with single power two circuits and current carrying mould (ESR-STCCM) has been put forward. The advantages of single power than two powers ESR process lies in lower appliance cost, simpler operation and no mutual interference of two powers. The ESR-STCCM process has two different types as shown in Figs. 1(a) (down power) and 1(b) (up power). Current through consumable electrode and current carrying mould is applied simultaneously in Fig. 1(a), however, they are opposite condition in case of Fig. 1(b). There are two par- allel loops in this technology. However, there is only one circuit for the conventional electroslag remelting process as shown in Fig. 1(c).

In order to simplify the calculation, some important hypotheses are made as follows:

(1) The process is considered as quasi-steady state;

(2) Induced magnetic field in the system has been ignored.

(3) Physical chemistry properties of steel and slag associates only with temperature.

(4) Latent heat released by solidification process will be introduced into heat transfer equation in the form of interior heat source.

(5) In the steel the Joule heating is negligible due to the low electric resistance of the steel.

(6) Consumable electrode tip is horizontal and constant, interface of slag and metal pool is a horizon surface.

\section{Mathematical Model and Boundary Conditions}

The electroslag remelting process is a very complex system, involving many physical phenomenons such as gravity, buoyancy, electromagnetic phenomena, fluid flow, heat transfer, and so on. The proper statement of the problem requires the definition of both the appropriate fluid flow equations and electromagnetic field equations. The basis descriptions of the process have been illustrated in many literatures. $^{6-10)}$

\footnotetext{
* Corresponding author: E-mail: dongyw@smm.neu.edu.cn

DOI: http://dx.doi.org/10.2355/isijinternational.ISIJINT-2016-066
} 

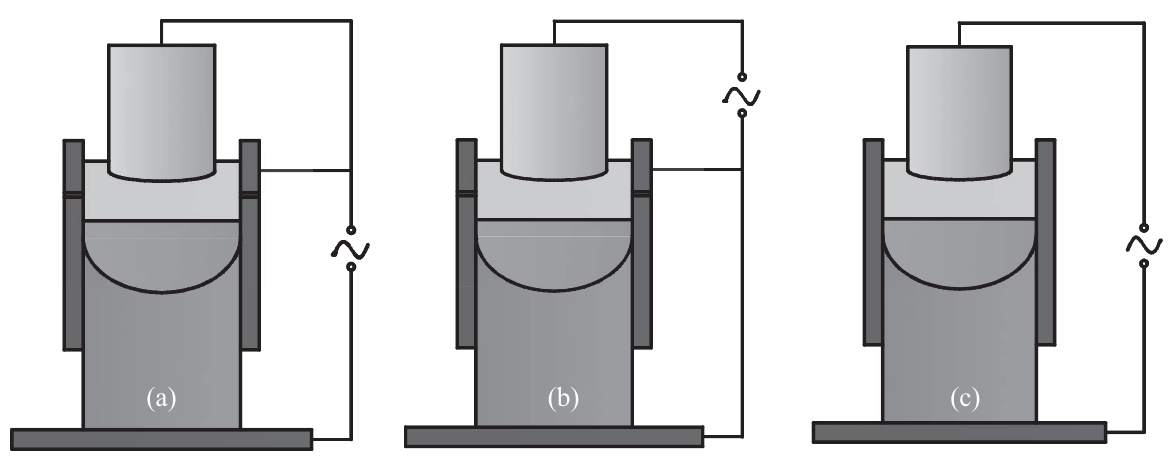

Fig. 1. Electroslag remelting: (a) down power, (b) up power, (c) conventional process.

\subsection{Governing Equations}

\subsubsection{Magnetic Field}

Usually, Maxwell's equations are used to calculate the current density distribution in the system and further the Joule heat density can be computed. ${ }^{7,9)}$

Ampere's law:

$$
\begin{array}{r}
\nabla \times H=J+\frac{\partial D}{\partial t} \\
\nabla \cdot B=0 \ldots \ldots \\
\nabla \cdot J=0
\end{array}
$$

Faraday's law:

$$
\nabla \times E=-\frac{\partial B}{\partial t}
$$

Current density distribution can be expressed as follow:

$$
J=-\sigma \nabla \varphi
$$

And then the Joule heat density can be achieved:

$$
Q_{j}=J \cdot J / \sigma
$$

Here, $E$ is electric field intensity, $H$ the magnetic field intensity, $B$ the magnetic flux density, $J$ current density, $\sigma$ the electrical conductivity of slag, $Q_{j}$ the Joule heat per unit volume, $\varphi$ the potential and $D$ the electric flux density.

\subsubsection{Fluid Flow}

Fluid flow, driven by electromagnetic force, gravity, buoyancy, etc., is an important phenomenon in ESR process. Electromagnetic force plays the major role in the fluid flow. The fluid flow in ESR process can be described by continuity equation and Navier-Stokes equations at axisymmetric cylindrical coordinate system: ${ }^{1-13)}$

$$
\begin{gathered}
\frac{\partial \rho}{\partial \tau}+\nabla \cdot v=0 \ldots \ldots \ldots \ldots \ldots \ldots \ldots \ldots \ldots \ldots \ldots \ldots \ldots \ldots \ldots \ldots \ldots \\
\frac{\partial \rho v}{\partial \tau}+\rho(v \cdot \nabla) v=-\nabla P+\nabla \cdot\left(\mu_{\mathrm{eff}} \cdot \nabla v\right)+\rho g+S_{S} \ldots
\end{gathered}
$$

Where $\rho$ is density, $v$ stands for velocity vector, $P$ is pressure, $g$ represents gravity acceleration, $\mu_{\text {eff }}$ stands for effective viscosity including molecular viscosity and eddy viscosity, $S_{S}$ is source term including electromagnetic force, buoyancy force and interfacial tension.

The electromagnetic force is obtained based on the fol- lowing equation:

$$
F_{\mathrm{loc}}=J \times B=\mu_{0} J \times H
$$

Here, $\mu_{0}$ denotes absolute magnetic permeability of vacuum, $F_{\text {loc }}$ the electromagnetic force, which is one of source term of fluid flow equation.

Turbulent viscosity is computed by $k-\varepsilon$ turbulence model. Standard $k-\varepsilon$ model has been used in practical engineering flow calculations since it was proposed by Launder and Spalding. ${ }^{14)}$ The original turbulence kinetic energy, $k$, and its rate of dissipation, $\varepsilon$, are obtained from the following transport equations:

$$
\begin{aligned}
& \frac{\partial}{\partial \tau}(\rho k)+\frac{\partial}{\partial x_{i}}\left(\rho k u_{i}\right) \\
& =\frac{\partial}{\partial x_{j}}\left[\left(\mu+\frac{\mu_{t}}{\sigma_{k}}\right) \frac{\partial k}{\partial x_{j}}\right]+G_{\mathrm{k}}+G_{\mathrm{b}}-\rho \varepsilon-Y_{M}+S_{k}
\end{aligned}
$$

$$
\begin{aligned}
& \frac{\partial}{\partial \tau}(\rho \varepsilon)+\frac{\partial}{\partial x_{i}}\left(\rho \varepsilon u_{i}\right) \\
& =\frac{\partial}{\partial x_{j}}\left[\left(\mu+\frac{\mu_{t}}{\sigma_{\varepsilon}}\right) \frac{\partial \varepsilon}{\partial x_{j}}\right]+C_{1 \varepsilon} \frac{\varepsilon}{k}\left(G_{\mathrm{k}}+C_{3 \varepsilon} G_{\mathrm{b}}\right)-C_{2 \varepsilon} \rho \frac{\varepsilon^{2}}{k}+S_{\varepsilon}
\end{aligned}
$$

In these equations, $k$ is kinetic energy of turbulence; $u_{i}$ is the velocity parallel to the wall, $\varepsilon$ represents dissipation rate of turbulence, $G_{k}$ denotes the generation of turbulence kinetic energy due to the mean velocity gradients, $G_{b}$ is the generation of turbulence kinetic energy due to buoyancy, $S_{k}$ and $S_{\varepsilon}$ are user-defined source terms. The quantities $\alpha_{k}$ and $\alpha_{\varepsilon}$ are the inverse effective Prandtl numbers for $k$ and $\varepsilon$, respectively. $C_{1 \varepsilon}, C_{2 \varepsilon}$ and $C_{3 \varepsilon}$ are constants. $\sigma_{\kappa}$ and $\sigma_{\varepsilon}$ are the turbulent Prandtl numbers for $k$ and $\varepsilon$, respectively.

Fluid flow of molten steel pool is ignored in this paper in order to simplify the calculation.

\subsubsection{Heat Transfer}

Heat transfer is one of the most important phenomena in the process of electroslag remelting, which determines the temperature distribution and solidification during ESR process. Heat transfer, can occur through conduction, convection, and radiation that is different for distinct region of electroslag remelting. The governing equations of thermal transfer for slag pool region can be expressed in the following form: 


$$
\begin{aligned}
& r \rho C_{p} V_{\mathrm{c}} \frac{\partial T}{\partial z}+C_{p}\left[\frac{\partial}{\partial z}\left(T \frac{\partial \psi}{\partial r}\right)-\frac{\partial}{\partial r}\left(T \frac{\partial \psi}{\partial z}\right)\right] \\
& =\frac{\partial}{\partial r}\left(K r \frac{\partial T}{\partial r}\right)+\frac{\partial}{\partial r}\left(K r \frac{\partial T}{\partial r}\right)+r S_{T}
\end{aligned}
$$

In which, $r$ is radius, $C_{p}$ represents specific heat of liquid slag, $V_{\mathrm{c}}$ stands for casting rate, $K$ is thermal conductivity, $\Psi$ is the stream function, $S_{T}$ is net heat generation rate in slag and it can be expressed as:

$$
S_{T}=Q_{\mathrm{j}}-Q_{\mathrm{d}} \chi
$$

$Q_{\mathrm{d}}$ is heat absorption rate by droplet in slag, $Q_{\mathrm{j}}$ represents Joule heat generation rate and $\chi$ stands for a position function.

At the same time, the thermal transfer equations of electrode, metal pool, mushy zone, and ingot region takes the following form at cylindrical coordinate:

$$
r_{i} \rho_{i} c_{P, i} v_{i} \frac{\partial T}{\partial z}=\frac{\partial}{\partial r}\left(K_{i} r \frac{\partial T}{\partial r}\right)+\frac{\partial}{\partial z}\left(K_{i} r \frac{\partial T}{\partial z}\right)+r S_{\mathrm{T}, i}
$$

Where $i$ is the different part incuding electrode, metal pool, mushy zone or ingot.

\subsection{Boundary Conditions}

\subsubsection{Magnetic Field}

A zero potential is imposed at slag/pool interface. At the same time, a higher potential is set at the slag/electrode contact interface for conventional ESR process. However, the same higher potential is imposed at the conductive part of current carrying mould for ESR-STCCM process. Horizontal magnetic field intensity at the upper surface of the electrode and the lower surface of ingot is assumed to be zero.

Magnetic field intensity of these positions including nonimmersed electrode lateral surface, free surface of slag pool, the lateral wall of ingot from upper surface of slag pool to bottom of ingot besides the conductive loop of current carrying mould can be described as follow:

$$
H=\frac{I_{i}}{2 \pi R_{i}}
$$

In which $i$ represents the relvant positon, $I_{i}$ the total current from center to $i$ position. The same description mentioned above can be applied to conductive loop of current carrying mould.

\subsubsection{Fluid Flow}

The free surface of slag pool is set as free slip interface. The interfaces of slag/current conductive part of mould, slag/insulating layer of mould, slag/non-conductive mould, slag/electrode and slag/metal are set as no slip interface.

\subsubsection{Heat Transfer}

Temperature is one of the most important boundary conditions. Liquidus temperature of metal is imposed at the lower surface of electrode. The peripheral boundary temperature of slag pool is the liquidus temperature of slag. Boundary conditon of heat transfer is very complex. The upper surface of slag pool exchange heat with air by natural convection as well as thermal radiation that related to the dimension from slag pool surface to furnace mouth in the
Table 1. Physical property parameter of steel and slag.

\begin{tabular}{ccc}
\hline Properties & Metal & Slag \\
\hline Density $/\left(\mathrm{kg} \cdot \mathrm{m}^{-3}\right)$ & 7500 & 2690 \\
Liquidus temperature $/ \mathrm{K}$ & 1623 & 1571 \\
Solidus temperature $/ \mathrm{K}$ & 1473 & - \\
Specific heat $/\left(\mathrm{J} \cdot \mathrm{kg}^{-1} \cdot \mathrm{K}^{-1}\right)$ & 720 & 837 \\
Viscosity $/\left(\mathrm{kg} \cdot \mathrm{m}^{-1} \cdot \mathrm{s}^{-1}\right)$ & - & 0.02 \\
Thermal conductivity $(\mathrm{s}) /\left(\mathrm{W} \cdot \mathrm{m}^{-1} \cdot \mathrm{K}^{-1}\right)$ & 16.72 & 10.45 \\
Latent heat of fusion $/\left(\mathrm{J} \cdot \mathrm{kg}^{-1}\right)$ & $2.1 \times 10^{5}$ & - \\
Magnetic permittivity $/\left(\mathrm{H} \cdot \mathrm{m}^{-1}\right)$ & $1.257 \times 10^{-6}$ & $1.257 \times 10^{-6}$ \\
\hline
\end{tabular}

Table 2. Model dimensions and process parameters.

\begin{tabular}{cc}
\hline Dimension and Parameter & Value \\
Voltage/V & 30 \\
Total current/A & 4500 \\
Electrode insert depth/mm $/ \mathrm{mm}$ & 120 \\
Electrode diameter/mm & 20 \\
Mould diameter/mm & 138 \\
Insulating layer thickness $/ \mathrm{mm}$ & 230 \\
Current conductive mould insert depth/mm & 2 \\
\hline
\end{tabular}

vertical direction and the dimension from mould wall to electrode surface. Heat transfer coefficient between cooling water and ingot surface or slag lateral surface is influenced by many factors such as slag skin, air gap and emission and so on. Determination of these parameters can be found in previous literatures.

\subsection{Numerical Solution of Governing Equations}

In the present work, all the furnace geometries are axisymmetric. The electrode-slag and slag-metal interfaces are assumed to be flat. The immersion depth of the electrode is $20 \mathrm{~mm}$, which remains constant during the entire simulation. Commercial software FLUENT as well as user-defined functions have been employed to solve these models established above. Direct current has been imposed in this work. In order to increase the precision of the model finer mesh has been applied for all kinds of interface including slag/ electrode interface, slag/mould interface, slag/metal interface and so on. A high alloy material has been employed in the model. The physical parameters of metal and slag are shown in Table 1. ${ }^{15)}$ Physical dimensions and process parameters are listed in Table 2.

\section{Results and Discussion}

\subsection{Magnetic Field and Velocity Distribution}

As a matter of fact, current carrying mould is an important progress in electroslag metallurgy. The process of single power two circuits ESR with current carrying mould will be more benefit for improving solidification quality than conventional ESR. In order to make clear the difference of these conditions, conventional ESR process will 
be used for comparison in this work. Figure 2 is current density distribution in slag pool. It can be seen that the current in the electrode is flowing from the electrode tip to the slag pool no matter what kinds of furnace mentioned in this work. Moreover, the current passing through electrode in Figs. 2(b) and 2(c) is larger than that of Fig. 2(a) of the same total current. Because the total current flows through the electrode in Figs. 2(b) and 2(c). Only partial current
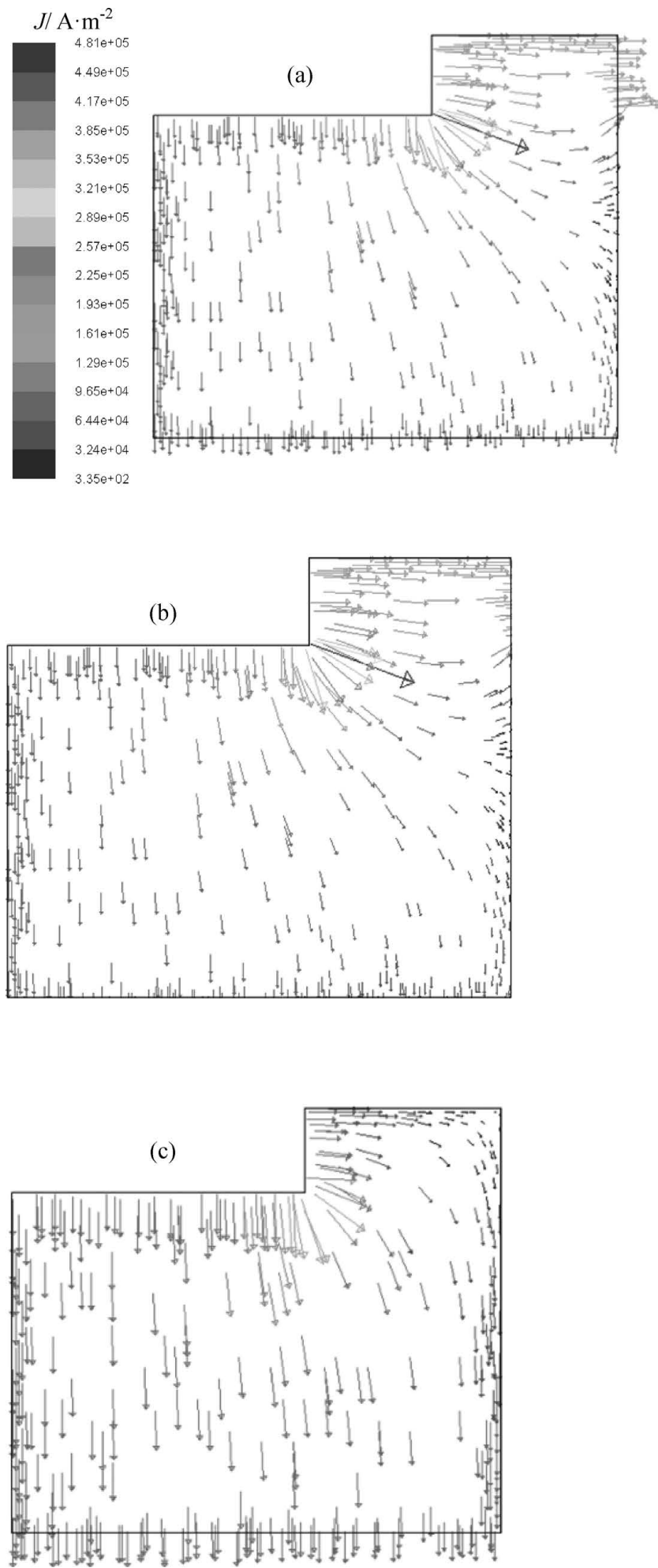

Fig. 2. Current density distribution in slag pool: (a) ESR-STCCM with down power; (b) ESR-STCCM with up power; (c) Conventional ESR. of total current flows through the electrode in Fig. 2(a). However, it is another situation in the slag-metal interface. The total current through slag-metal interface returns the transformer in Figs. 2(a) and 2(c). Only partial current of total current flows through the slag-metal interface returns the transformer in Fig. 2(a) because some of them return the transformer through current carrying mould. The larger current density lies in only the position of electrode corner in Fig. 2(c), yet the current density is also larger in the posi-

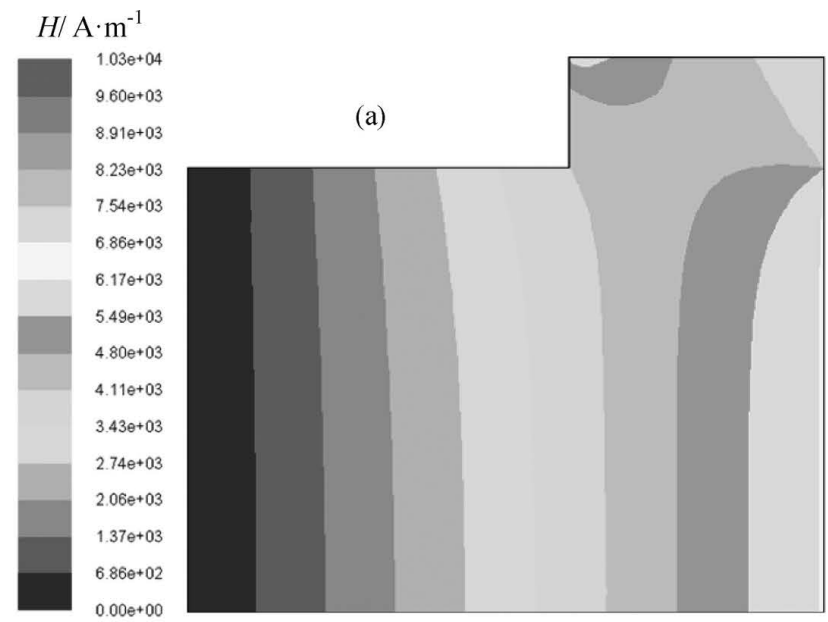

(b)

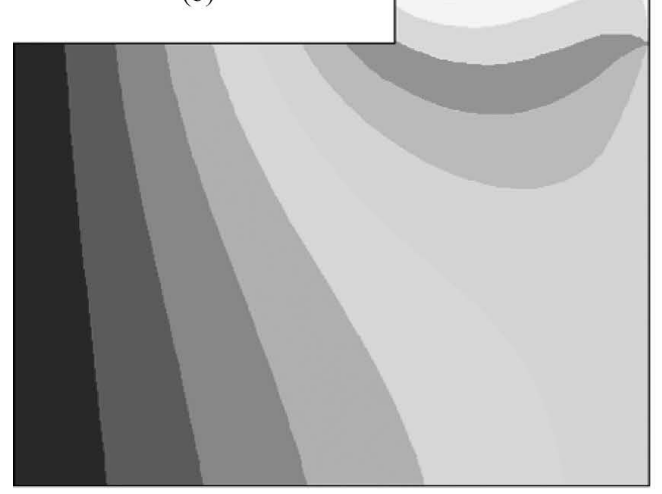

(c)

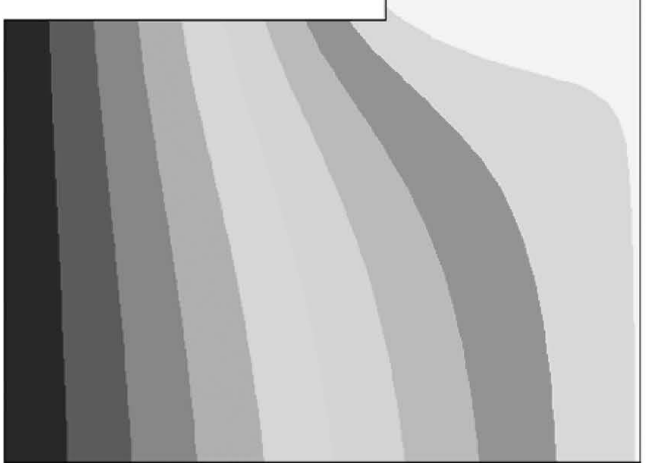

Fig. 3. Magnetic field intensity: (a) ESR-STCCM with down power; (b) ESR-STCCM with up power; (c) conventional ESR. 
tion of conductive part of current carrying mould besides the electrode corner in Figs. 2(a) and 2(b).

Special current distribution leads to different magnetic field and velocity distribution as shown in Figs. 3 and 4. In the medium, the magnetic field intensity is usually defined as:

$$
H=\frac{B}{\mu_{0}}-M
$$
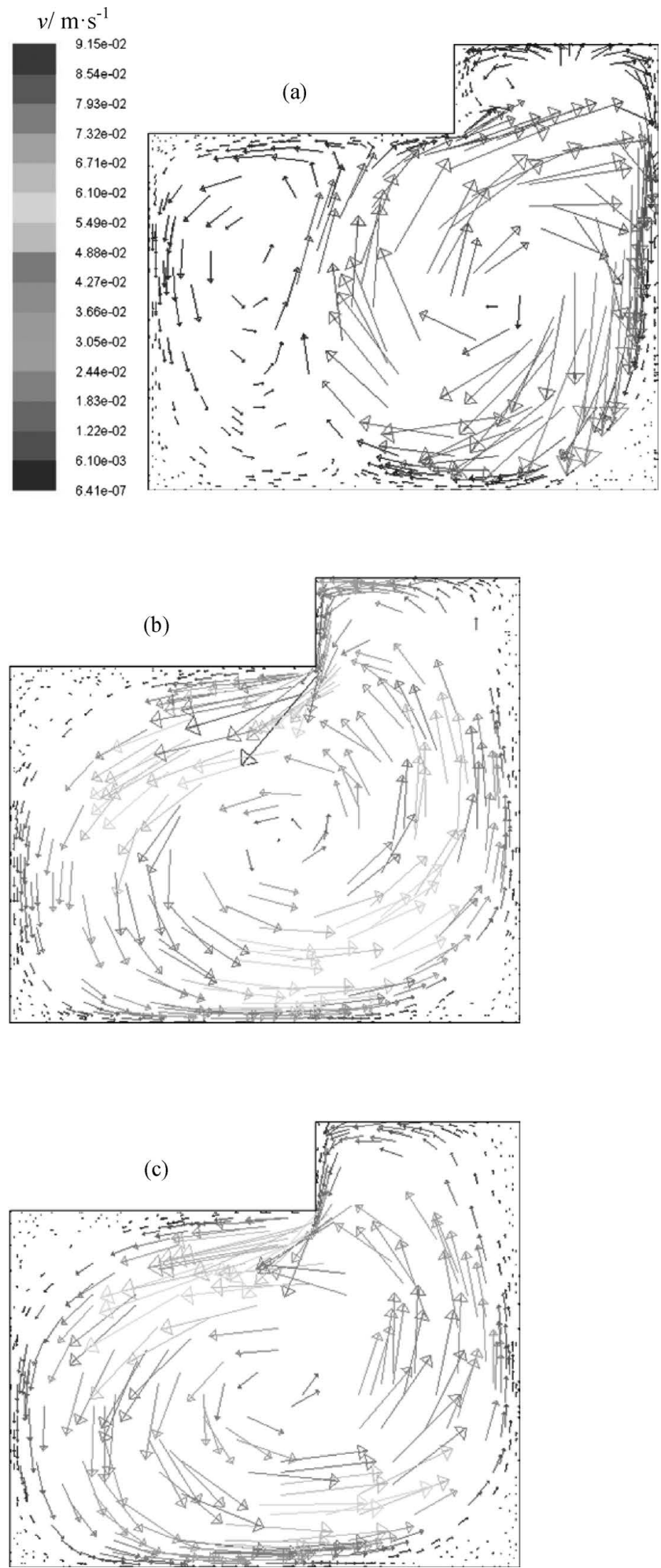

Fig. 4. Velocity distribution in slag pool: (a) ESR-STCCM with down power; (b) ESR-STCCM with up power; (c) conventional ESR.
In which, $\mathrm{M}$ is the magnetization intensity of medium. It can be seen that magnetic field intensity is proportional to magnetic flux density. Usually the magnetic flux density can be described as the following equation.

$$
B=\frac{\mu_{0} I}{2 \pi r}
$$

In the equation two variations including current and

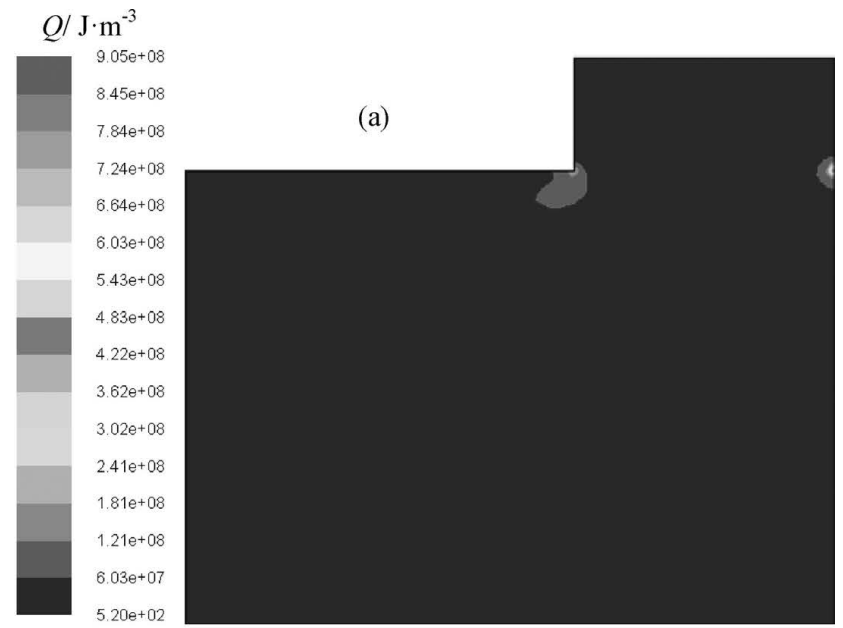

(b)

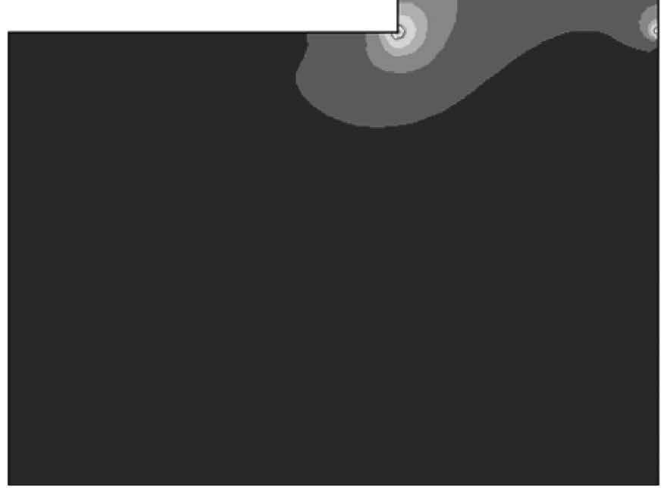

(c)

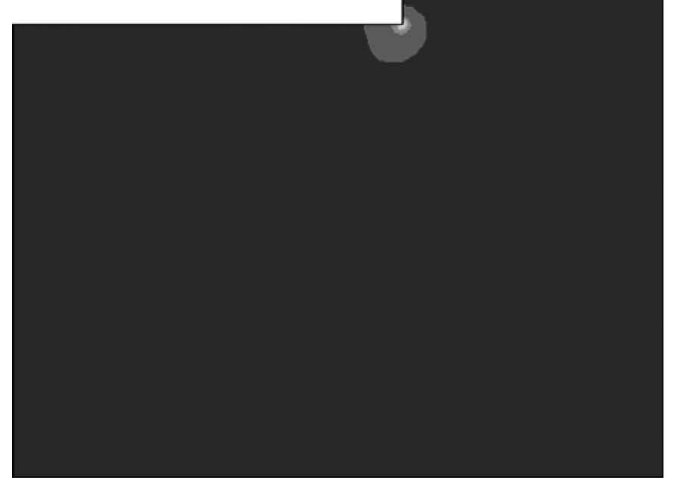

Fig. 5. Joule heat density distribution: (a) ESR-STCCM with down power; (b) ESR-STCCM with up power; (c) conventional ESR. 
distance from center will affect magnetic field intensity. Results indicate that magnetic field intensity is small in the center of slag pool due to less current in this location. Magnetic field intensity increases with the increasing of distance from center in the slag-metal interface. Obviously, the influence of current is larger than distance. However, magnetic field intensity near the position of lateral electrode surface immersed in slag pool is almost the largest in Figs. 3(b) and 3(c) because total current flows from electrode. In addition, it can be seen that magnetic field intensity between lateral electrode and mould wall for ESR-STCCM and conventional ESR. Larger magnetic field intensity lies in the positions of electrode-top slag surface and slag-lower area of conductive part of current carrying mould for ESRSTCCM process. Moreover, the contour of magnetic field intensity just likes the current direction as shown in Fig. 2.

The buoyancy force and the Lorentz force are the most important forces, which affect the motion of fluids. As observed in Fig. 4 that flow of slag pool is obvious different for different furnace type. Almost three vortexes can be noticed in Fig. 4(a). Only there is a big vortex in Figs. 4(b) and 4(c), moreover, they are all counterclockwise circulation mainly induced by electromagnetic force. Even so, the velocity of slag flow of ESR-STCCM with upper power is higher than conventional ESR process. The biggest vortex, oriented in the clockwise direction, may be induced by buoyancy in Fig. 4(a) because the slag will descend due to the density increase and temperature decrease, which is due to the effect of cooling water in mould. However, a counterclockwise circulation can be noticed beneath the electrode, which is mainly caused by electromagnetic and falling metal droplets. In addition, there is a small vortex in the corner between lateral electrode and mould wall. It can be deduced that it should be the common impact of Lorentz force and buoyancy force.

\subsection{Temperature Distribution in Liquid Slag Pool}

Current density plays an important role in Joule heat distribution. It can be seen from Fig. 5 that Joule heat density in the position of electrode corner is larger for any ESR process in this work. Moreover, the lower area of conductive part of current carrying mould has also larger Joule heat density for ESR-STCCM process. There are two larger

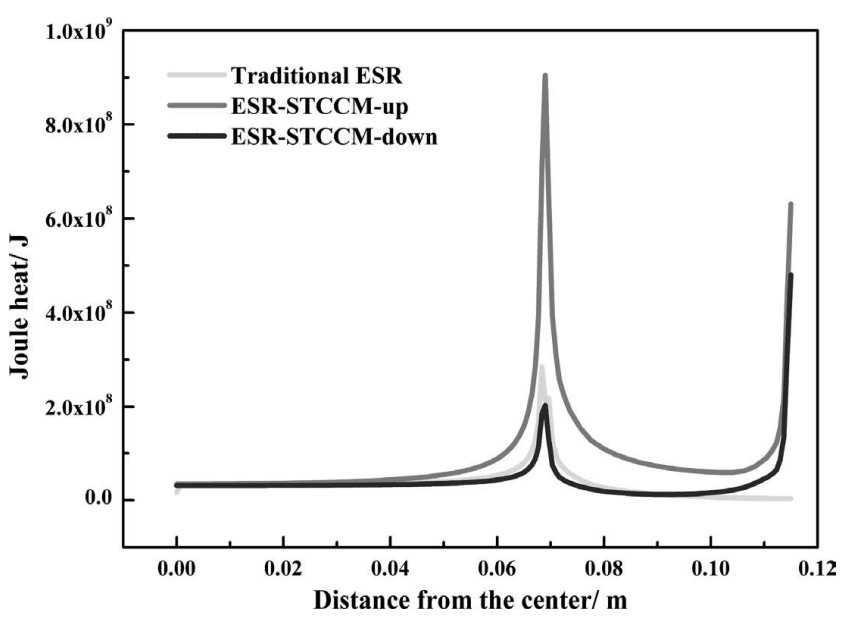

Fig. 6. Joule heat density of electrode tip position from center to edge in horizontal direction.
Joule heat density positions in ESR-STCCM process, which is obviously different from one larger Joule heat position in conventional ESR process.

In order to compare the difference of different ESR type mentioned in this work, the Joule heat density in the electrode tip position where is from center to edge, has been extracted as shown in Fig. 6. As observed that the largest Joule heat density is up to $9.04 \times 10^{8} \mathrm{~J} / \mathrm{m}^{3}$ for ESR-STCCM

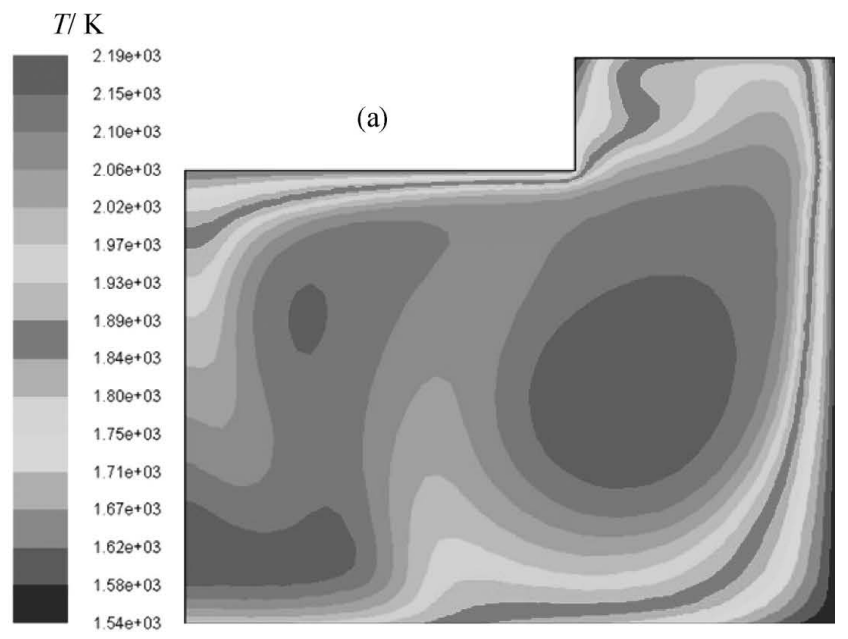

(b)

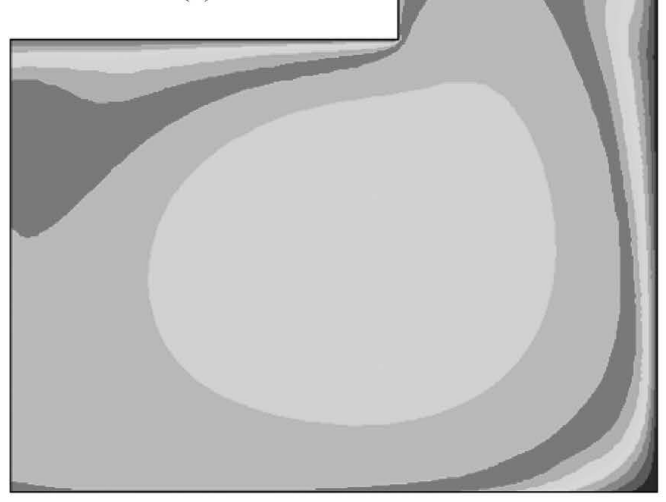

(c)

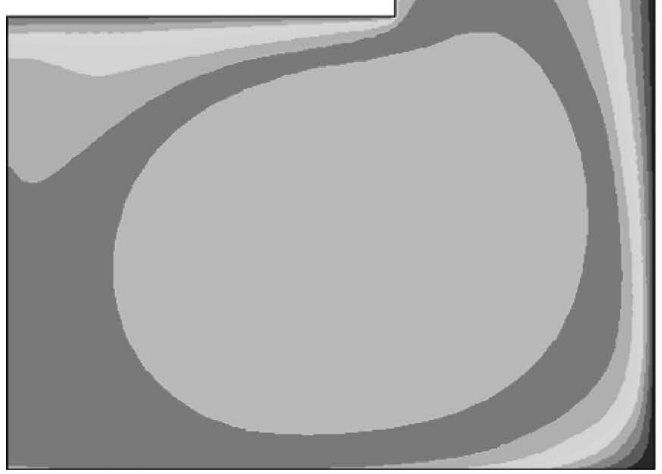

Fig. 7. Temperature distribution in slag pool: (a) ESR-STCCM with down power; (b) ESR-STCCM with up power; (c) conventional ESR. 
with up power; however, it is only $4.80 \times 10^{8} \mathrm{~J} / \mathrm{m}^{3}$ for ESRSTCCM with down power. Additionally, the gradient of Joule heat density is very significant in the position of electrode corner and edge of current carrying mould.

Special flow and Joule heat distribution in slag result in different thermal field as shown in Fig. 7. There are multiple high temperature regions in ESR-STCCM with down power; however, there is only one high temperature region in ESR-STCCM with up power and conventional ESR process. The shape of thermal field is similar in Figs. 7(b) and 7(c), but the area of high temperature zone in Fig. 7(b) is wider than that in Fig. 7(c). Accordingly, the thermal field in Fig. 7(b) will be more homogeneous than that in Fig. 7(c). Results also indicate that high temperature zone center is below the lateral electrode in Figs. 7(b) and 7(c).

In addition, the temperature in Figs. 7(b) and 7(c) at the lower part near mould wall in slag pool is higher than that in Fig. 7(a), which contributes to achieve homogeneous thermal field distribution in slag pool. It can also be observed that there is another high temperature zone below electrode near slag-metal interface, which will increase the slag-metal interface temperature of adjacent area as shown in Fig. 8. The center temperature of ESR-STCCM with down power is obviously higher than other ESR process. Moreover, the temperature difference between the highest and the low-

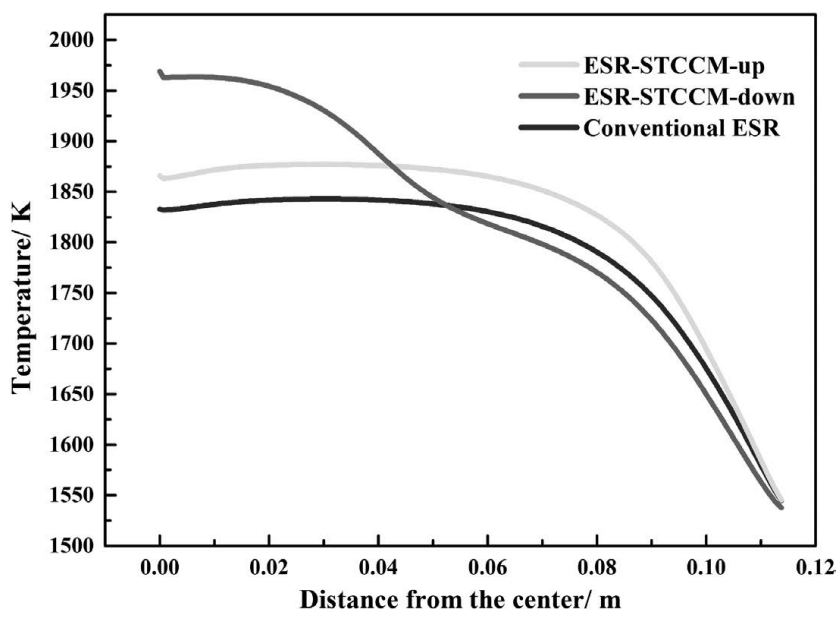

Fig. 8. Temperature distribution of slag-metal interface. est temperature is the largest for ESR-STCCM with down power process among the three ESR process. On another, the temperature is the highest at the same distance from center in horizontal direction for ESR-STCCM with up power process. It contributes to obtain shallow molten steel pool.

\subsection{Molten Steel Pool}

All the time, the shape and depth of molten steel pool has been considered as an important material solidification quality evaluation standard. Therefore, ingot temperature distribution is one of the most important results in simulating the ESR process. Reducing the depth of molten steel pool and improving ingot solidification quality are the final aim for developing new process in electroslag metallurgy process.

Figure 9 is the liquid fraction in ingot. It can be seen the molten steel pool is the deepest in conventional ESR, however, it is the shallower in ESR-STCCM with up power among the three processes. In order to find the abstract difference in dimension, some data have been extracted from this figure. Figure 10 shows the liquidus and solidus line position for different ESR process. Just as mentioned before, the molten steel pool of ESR-STCCM with up power is the shallowest and it is approximate for conventional ESR and ESR-STCCM with down power. In fact, the height of mol-

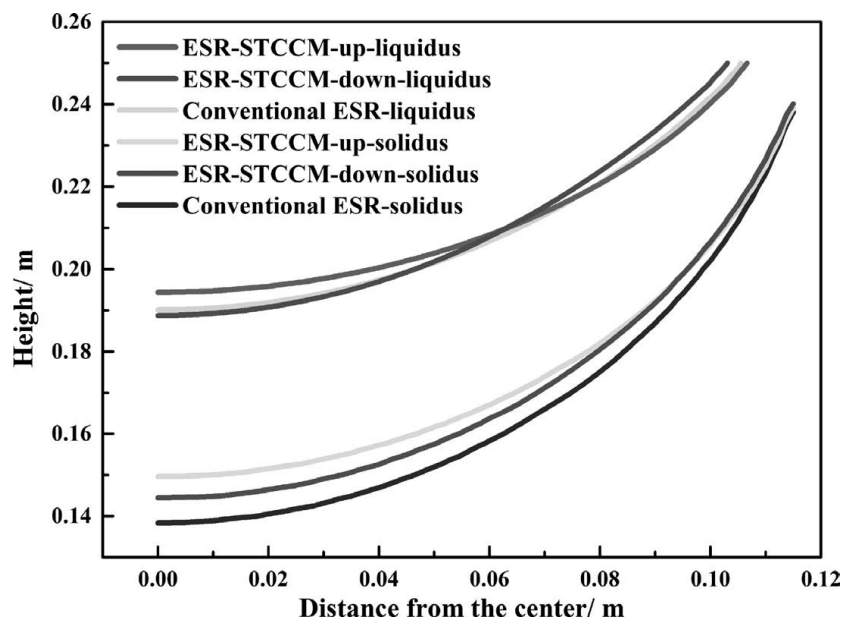

Fig. 10. Liquidus and solidus line position of different ESR process.
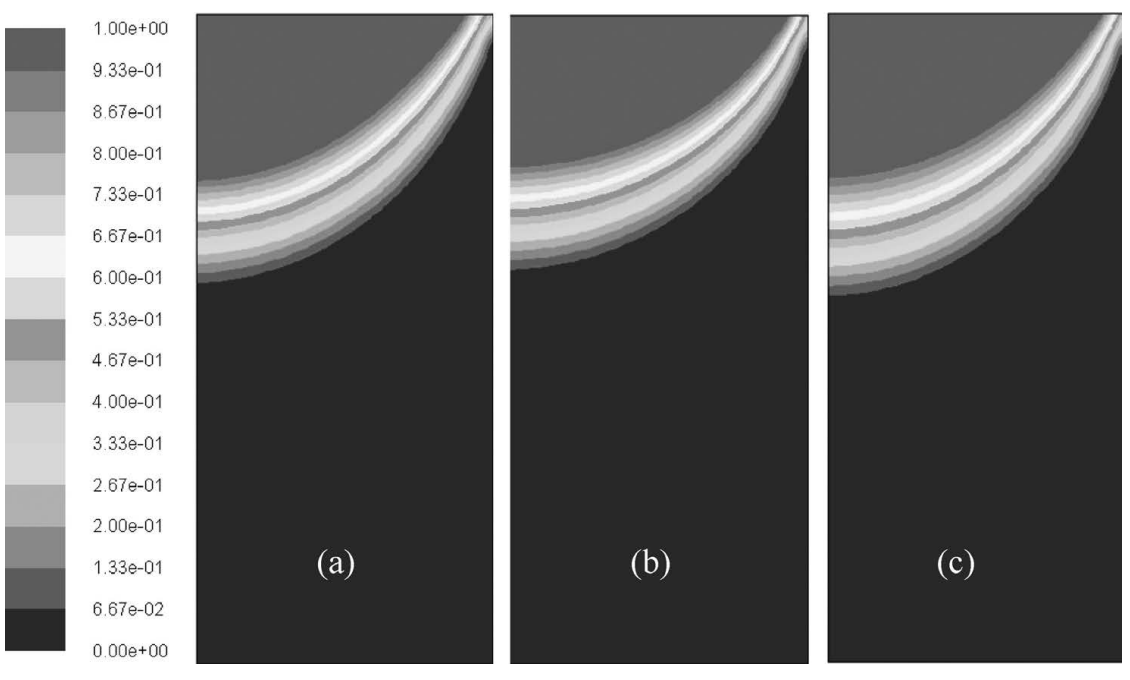

Fig. 9. Liquid fration in ingot: (a) ESR-STCCM with down power; (b) ESR-STCCM with up power; (c) conventional ESR. 


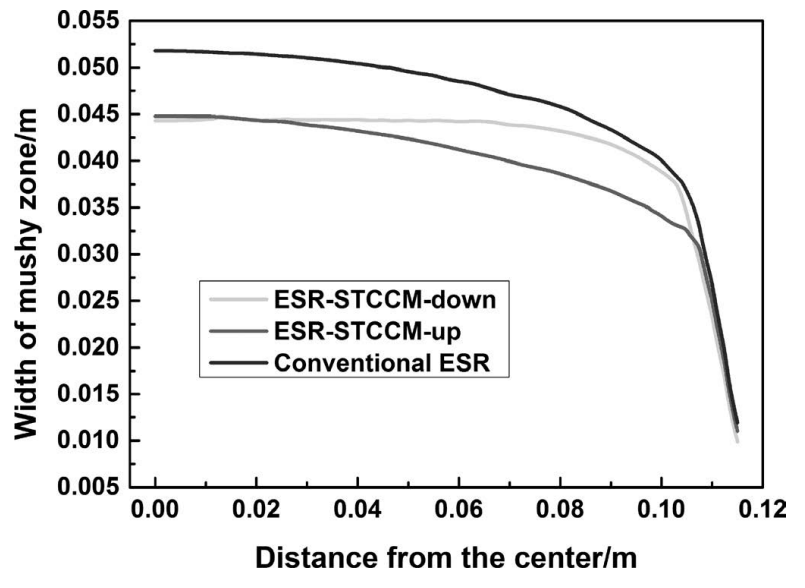

Fig. 11. Variety of mushy zone width.

ten steel pool near mould wall affect the ingot surface quality. The higher molten steel pool temperature near mould wall is, the better ingot surface quality is. Therefore, it is not difficult to draw the conclusion that ESR-STCCM with up power is favor of obtaining ingot with smooth surface quality according to the results mentioned above.

In addition, as it is well known, the width of mushy zone plays an important role in steel inner solidification quality. Figure 11 illustrates the width of mushy zone. It is almost the same width of mushy zone in the center for ESRSTCCM process. Width of mushy zone of ESR-STCCM with up power decreases faster than that of ESR-STCCM with down power with the distance increase from center. It can be inferred that ESR-STCCM with up power process will achieve shorter local solidification time and contribute to control the solidification quality. The mushy zone of conventional ESR process is obvious wider than that of ESR-STCCM process. Therefore, conventional ESR process for controlling material prone to segregation is worse than ESR-STCCM process.

\section{Conclusions}

ESR-STCCM with different current circuits including up power and down power as well as conventional ESR process has been investigated by simulation in this paper. Some conclusions can be draw as follows:
(1) Various physical fields including electromagnetic field, fluid flow, and thermal field and so on for ESR-STCCM process are different from conventional ESR process.

(2) Slag near conductive part of current carrying mould generates Joule heat due to the current pass.

(3) Molten steel pool for ESR-STCCM is shallower than conventional ESR process especially for ESR-STCCM with up power.

(4) ESR-STCCM is more suitable for controlling steel inner solidification quality than conventional ESR process.

\section{Acknowledgments}

This project supported by Fundamental Research Funds for Central University of China with the grant No. 150202003. Also, this project supported by Joint Research Fund of National Nature Science Foundation of China and Baosteel Group Corporation with the grant No. U1360103 and supported by the National Nature Science Foundation of China with the grant No. 51274266.

\section{REFERENCES}

1) L. B. Medovar, A. K. Tsykulenko, A. V. Chernets, B. B. Fedorovskii, V. E. Shevchenko, I. A. Lantsman, T. F. Grabovskii, V. I. Us and V. L. Petrenko: Adv. Spec. Electrometall., 16 (2000), 193.

2) A. K. Tskulenko, I. A. Lantsman, L. B. Medovar, A. V. Chernets, V. E. Shevchenko, B. B. Fedorovskii, T. F. Grabovskii and V. I. Us: Adv. Spec. Electrometall., 16 (2000), 141.

3) H. Holzgruber, W. Holzgruber and A. Scheriau: Proc. Int. Symp. Liquid Met. Proc. Cast., PRES de 1'Université de Lorraine, Nancy, France, (2011), 41.

4) Y. W. Dong, Z. H. Jiang, L. Medovar, G. Stovchenko, X. F. Zhang, X. M. Zang and X. Deng: Steel Res. Int., 84 (2013), 1011.

5) F. B. Liu, Z. H. Jiang, H. B. Li, X. Geng, H. Feng and X. M. Zang: Ironmaking Steelmaking, 41 (2014), 790.

6) A. H. Dilawari and J. Szekely: Metall. Trans. B, 9 (1978), 77.

7) B. Hernandez-Morales and A. Mitchell: Ironmaking Steelmaking, 26 (1999), 423.

8) K. M. Kelkar, J. Mok, S. V. Patankar and A. Mitchell: J. De Physique IV Dec., 12 (2004), 421.

9) M. Choudhary, J. Szekely, B. I. Medovar and Yu. G. Emelyanenko: Metall. Trans. B, 13 (1982), 35.

10) V. Weber, A. Jardy, B. Dussoubs, D. Ablitzer, S. Rybéron, V. Schmitt, S. Hans and H. Poisson: Metall. Mater. Trans. B, 42 (2009), 271

11) M. Choudhary and J. Szekely: Metall. Trans. B, 3 (1980), 439

12) J. H. Wei and Y. L. Ren: Acta Metall. Sin., 2 (1995), 51.

13) Z. H. Jiang: Mathematical Simulation and ExperimentAanalysis of Tthermal Ttransfer for ESR. Shenyang: Northeastern University Press, (1986), 168

14) B. E. Launder and D. B. Spalding: Lectures in Mathematical Models of Turbulence, Academic Press, London, England, (1972), 90.

15) M. K. Kanchan and V. P. Suhas: Proc. Int. Symp. Liquid Met. Proc. Cast., ASM International, OH, (2005), 137. 